\title{
The Changing Role of Scientific Experiment
}

\author{
Peeter Müürsepp \\ Department of International Relations, Tallinn University of Technology
}

Practical realism is focused on the problem of how science really works. In the case of physics and chemistry, experiment is the centrepiece of scientific practice. The rapid development of contemporary natural science does not leave the experiment unaffected. The classical experiment is normally applied only to systems that can be considered structurally stable, repeatability being the key feature. After the introduction of the theoretical basis of irreversibility by Ilya Prigogine the essence of the experiment changed. The strict requirement of repeatability has to be dropped. It will be discussed, whether the change is big enough for calling it revolutionary. There are means to update the understanding of the experiment by applying the experimental settings. The material experiment will probably be with us forever but its position on the scientific landscape will be shifted.

Keywords: experiment, non-repeatability, irreversibility, laboratory fictions, Prigogine, Rouse

\section{Introduction}

It can be considered a well formed position that philosophy of science has been narrowing itself down for quite a while. It has been theory focused. Besides, some philosophers of science have been trying to tell the scientists how science should work. In the framework of such intellectual reality, there is nothing to be surprised of Richard Feynman's famous dictum that philosophy of science is as useful to scientists as ornithology is to birds. It is rather the philosophers of science who are responsible for such attitude to appear rather than "orthodox" physicists like Feynman. Fortunately to philosophers it is quite easy to argue with Feynman claiming that ornithological knowledge has actually worked to the benefit of birds at least in two ways, one real and the other hypothetical. First, the growth of knowledge in ornithology has enabled to work out more and more effective measures for

Corresponding author's address: Peeter Müürsepp, Tallinn University of Technology, Akadeemia tee 3, Tallinn 12618, Estonia. Email: peeter.muursepp@tseba.ttu.ee. 
bird protection. Second, the birds themselves should be able to implement the knowledge produced by ornithologists. They are not so far, but could be. It is an analogous situation as with some scientists who are simply unable to make sense of philosophy. Their view seems to be just something like general naïve realism, taking philosophy as a popular generalization of the results of scientific research.

In the light of the debates like the one just addressed, focusing on how science actually works, looks a real innovation in the philosophical tradition of studying science. Still, scientists might claim that they who are actually engaged in practical research know that better. But again, who knows better, how the birds actually fly, the ornithologists or the birds themselves? Well, they have different knowledge about the issue. One is not necessarily better than the other.

Obviously, practical realism does not start from an empty spot. There is the Marxist understanding of practice. There is the American pragmatism. Practical approach to science was clearly attempted by Thomas Kuhn. It is the advance of the philosophy of chemistry, however, that has given a real impetus to the tradition and most importantly added the innovative flavour that was not there before.

The Estonian philosopher of chemistry Rein Vihalemm has characterised practical realism by five main theses in several papers:

(1) science does not represent the world "as it really is" from a God's eye point of view;

(2) the fact that the world is not accessible independently of theoriesor, to be more precise, paradigms (practices) - developed by scientists does not mean that Putnam's internal realism (or social constructivism) is acceptable;

(3) science as a theoretical activity is only one aspect of it (of sciences) as a practical activity whose main form is scientific experiment which in its turn takes place in the real world, being a purposeful and critically theory-guided constructive, manipulative, material interference with nature;

(4) science as practice is also a social-historical activity which means, among other things, that scientific practice includes a normative aspect, too, and that means, in its turn, that the world as it is actually accessible to science is not free from norms either;

(5) though neither naïve nor metaphysical, it is certainly realism as it claims that what is "given" in the form of scientific practice is an aspect of the real world. (Vihalemm 2011, 48). 
The theses are mostly not about the experiment. Still, if anyone speaks about anything practical in science then the first intuition is normally directed to the experiment. Obviously, the approach is much broader than that. Practicing science does not necessarily mean experimenting after all. The experiment is just one, although a very important, element in science as a practical activity. However, if we take the stand that there are changes in practicing science happening over time then experiment most probably becomes affected as well. But does its essence change? Or if it does, can the new activity be called experiment any longer? The central claim of the current paper is that the essence of experiment has changed over the last decades and its role in natural science today is not the same as it was half a century ago. This concerns regular laboratory experiments, not necessarily thought experiments or computer experiments.

\section{The Traditional Repeatable Experiment}

The traditional material experiment is interference into nature. It disturbs natural settings. However, it has been believed that experimental justification of a theory of natural science is absolutely mandatory. Without experimental proof we would be trapped in metaphysics like in the cases where (classical) experiment in the traditional sense cannot be set up at all as in the case of studying ethical or religious issues or metaphysics itself. The traditional material scientific experiment does not just have to be possible but one has to be able to repeat it. Needless to say, the results should not differ too much. This is an important, even crucially so, detail. How much is too much? The answer is vitally important. It will enable us to determine whether we are dealing with a repeatable experiment or not. Obviously, this is not the only issue we can apply for drawing the line. We need to analyse the problem from two different methodological points of view. This is because from the subjective impression formed by human sense experience, strictly speaking nothing in this world is repeatable. Scientific theories, on the other hand, have been based on the idea of reversibility that has set the theoretical basis for the claim of the repeatability of experiments for centuries neglecting the role of the arrow of time altogether. For instance, concerning complex systems, one can state: "While life-less, complex systems are irreversible because of their complexity and, hence, not repeatable, living systems are reproduced by irreversible copy-reproduction and by coding. This mode of reproduction results of necessity in an arrow of time of growth and increasing complexity with death as its antagonist, and in obligatory asymmetry" (Walker 1983, 806).

Let us take a deeper look into the essence of the repeatability of experiments using a selection of approaches and concepts that are known in phi- 
losophy of science. For instance, the French mathematician and philosopher René Thom has claimed that all experimental science is based on the conception that experiments will be repeatable, stressing that saying that an experimental result is "not repeatable" is to suggest that the result is not true, at least a mistake, and perhaps even a fraud (Thom 1975). What could be worse than that for a researcher to hear? The critics say that this attitude alone has done a lot of harm to contemporary natural science preventing it from implementing novel research approaches and focuses that could have worked for a more efficient advancement of science than we have seen so far. However, this is the topic for the next chapter. On the other hand, the requirement of repeatability has helped to secure the necessary rigour of scientific research.

What does repeatability really mean? As it was stated above already, nothing in the empirical world has ever been repeatable in the direct meaning of the word. Even the Holy Bible recognizes that there is nothing new under the Sun, everything repeats, but in a new way (my emphasis-P.M.). It has been stressed by René Thom and several others that repeatability depends on whether you can duplicate the initial and surrounding preconditions precisely enough. We cannot expect the results to be the same if the pre-conditions are not the same. However, the pre-conditions cannot possibly be the same ever. Everything in the physical world is under constant change as far as representation of the initial conditions is concerned. It has mistakenly been believed that progress in building better and better measurement devices will cause this problem to disappear. This is not going to happen. The problem of initial conditions is not a problem of measurement. It is rather a problem of representation. We can elaborate the precision of the preconditions for ever adding new decimal digits to the measurement results as the devices progress. Absolute precision cannot be achieved because it does not exist in the world that is constructed like ours.

The question, how much is enough, or rather how much is not too much, meaning the difference in final results, will stay with us forever. What is the measure of repeatability? As a matter of fact, by and large, the answer to this question is a matter of convention, depending on how high precision are we looking for. Still, the issue is more complicated than that. Higher precision in the initial conditions does not necessarily guarantee the same for the results. This has been known for at least half a century already (if we do not consider Henri Poincarés calculations in early XX-th century the results of which the great mathematician himself was not sure of), after Edward Lorentz's famous computer-based studies of the weather patterns that led to the discovery of the so-called butterfly attractor. It may be that Lorentz had a computer that did not function properly. Many similar computer based experiments, how- 
ever, have been done after that. There is sufficient consistency with Lorentz's results for claiming that the computer cannot be "blamed" for the discovery of the butterfly effect. It has been proved that there can be wide differences in the degree of sensitivity to the inevitable, but sometime negligibly small, differences in pre-conditions. Some physical situations as well as some combinations of equations are highly susceptible to very small differences in initial conditions. Classical science has quite successfully managed to avoid studying such phenomena. Actually, they were very difficult to deal with before the age of the computers began. But there is no rational excuse for that avoidance any more.

It cannot be reasonably denied that there is consistency between the results of numerous computer simulations run by researchers and behaviour of the corresponding material structures. There are many computer simulations of physical processes that play an important role in the development and understanding of physics-based mathematical models. Some examples include, i.e. the cylinder deformation application or the reference distribution variable selection performance of simulated examples of varying complexity, not to speak of the numerous models developed in the scope of chaos theory

Obviously, the computer simulations broaden the scope of understanding of the material experiment beyond their regular reach. However, this has been done at the cost of loosening the link between the calculations done by the computer and material reality. Therefore, even the experiment in physics is not necessarily embedded in material structures any more. Computers are taking over because scientific research has gone beyond the reach of human sense perception and even imagination based on sense perception.

Again, it is René Thom who has argued that scientists have historically shied away from all those phenomena that have too great a degree of sensitivity to initial conditions (Thom 1975). This is true. However, it is not clear whether Thom himself was really trying to break through this old attitude or rather to strengthen it by means of proposing a new flexible approach to fit into the traditional framework. Here, we mean the conception or property of structural stability. The conception, initially a mathematical one, has been elaborated by Thom at length in his Structural Stability and Morphogenesis, the French original of which was published in 1972 and translated into English in 1975. An analysis of the conception can be found in my book (Müürsepp 2010). A system can be called structurally stable when it does not change qualitatively under the influence of small scale fluctuations. It is true that classical science avoids everything that is not structurally stable. Trying to break through this barrier René Thom normally still retains the traditional thinking of a classical scientist. It was Ilya Prigogine who man- 
aged to turn the tables. It is his approach that is the core of the non-classical view on science including the experiment itself. We shall address the Prigoginian ideas in more detail below.

As our analysis belongs to the frames of practical realism, we cannot avoid referring to the conception of $\phi$-science presented by Rein Vihalemm (2007). By $\phi$-science Vihalemm means a theoretical model of science proper. Physics is definitely the closest discipline to the model but does not coincide with it. The model is still a model, not any kind of specific branch of science itself. It is a steady methodological framework. Each and every branch of science changes constantly. According to the model of $\phi$-science, it is just the so-called constructive-hypothetico-deductive type of cognition that can be the basis of science proper. The researcher constructs the object of research for herself and does not study directly what nature provides. However, we cannot say that it is not reality that is being studied. It is a type of reality, the scientific one. There is also a good chance that it is not too far from reality concerning the world out there as the researcher still constructs her object not neglecting data obtained through sense experience by means of systematic observation. But we cannot be sure because we do not possess the God's eye. It is more complicated in the cases where we do not have observational acquaintance. There additional models have to be brought in.

Obviously, it is more convenient to set up an experiment while studying a constructed object than a natural one. Actually, this is the only way we can set up a repeatable experiment at all. Thus, classical repeatable experiment is a constructed situation. Science proper can work with such situations only. Now, we can add another point here. Only structurally stable phenomena can be studied by $\phi$-science. It is just the structurally stable phenomena that can be tested by means of classical repeatable experimentation.

What about quantum mechanics from the experimental point of view? Normally, it is considered to be one of the core examples of non-classical science. It is true, that statistics is present more clearly than in classical science and we necessarily have to consider statistical means when speaking about either initial conditions or results of a process. But repeatability is still required. Even more so, because the mean of a large number of experimental results have to be considered in order to be able to draw a conclusion. The situation studied in quantum mechanics is still an idealized one. The whole approach is based on reversible theoretical considerations, i.e. the Schrödinger equation. Actually, we are still in the classical context here. It is so concerning both theoretical and practical (experimental) considerations. Still, we cannot deny the fact that quantum theorists (and experimenters) fully acknowledge the fact that setting up an experiment and applying measurement breaks the natural sequence of events. 


\section{Making Sense of the New (Non-Repeatable) Experiment}

Classical natural science takes reversibility as an inevitable premise of any rigorous research. As we noted above, the same is basically true about quantum theory as well. There is no difference when we consider the other flagpole of the so-called non-classical science, namely relativity theories. The great Albert Einstein did not consider time to be anything else than an illusion. Why abandon the requirement of repeatability of experiments in the situation where the scientific scene seems to be in full support of the idea that the irreversibility of worldly phenomena is just an illusion, a subjective impression based on the limited capacity of our senses? If time does not matter, we can repeat everything we need as many times as we like and fine tune the result. This would not necessarily work in an ever changing irreversible world.

Before going on with the real non-classical approach that was brought about by Ilya Prigogine and Hermann Haken we have to pay a tribute to an exceptional thinker of late XIX-th early XX-th century. This is Ludwig Boltzmann who was on the right track while trying to introduce the evolutionary approach into physics. His main ideas necessarily involved the conception of irreversibility. Here is the moment in the history of science where the concept of the arrow of time finds its true place. Unfortunately, Boltzmann backed off after coming under severe criticism of opponents. It seems he never regained his mental stability after this debate. Boltzmann's theoretical considerations directly lead to the idea of the non-repeatable experiment. He was very close to getting there.

Boltzmann's main problem concerns directly a principle that has a close connection to experimental research undertaken in the XX-th century by Bénard, Belousov and Zhabotinsky, etc., namely the second law of thermodynamics. Boltzmann interpreted the second law as a practical, rather than a theoretical principle. He tried to introduce irreversibility into dynamics but failed to extend it into thermodynamics.

Boltzmann got trapped into a dramatic situation. He started to believe that in order to understand nature we have to include evolutionary features and that irreversibility, as defined by the second law of thermodynamics, was decisive here (Prigogine 1997, 21). But Boltzmann was also heir to the tradition of classical dynamics. He realized that giving a microscopic meaning to the arrow of time is crucial. Boltzmann had to choose between the idea that physics had to understand becoming and his belief in the traditional role of dynamics. The attempt of Boltzmann to introduce irreversibility on the basis of trajectories was hopeless as we know today. "As Henri Poincaré noted, explaining irreversibility in terms of trajectories that are time-reversible processes, however numerous, appears to be a purely logical error" (Prigogine 
1997, 21). Boltzmann's interpretation was probability-based. He managed to make the macroscopic character of our sense observations responsible for the irreversibility that we observe. Thus, irreversibility remained not a basic law of nature but merely a consequence of the approximate character of our observations. The revolution was postponed.

Eventually, the revolution was made possible by Ilya Prigogine. For Prigogine irreversibility becomes a fundamental theoretical fact. He postulated the second law of thermodynamics as a principle of selection leading to the breaking of time symmetry (Näpinen and Müürsepp 2002, 467). The new physical and mathematical concepts had to be created just for this reason. The breaking of time symmetry is an inner quality explained by the time operator. Thanks to the introduction of the operator it is possible to explain the inner breaking of time symmetry simultaneously in mechanics, quantum physics and relativity theories. The word 'inner' means that the breaking of time symmetry is not forced by new interactions (Näpinen and Müürsepp 2002, 467). The time operator is closely connected to the microscopic entropy operator. Through non-canonical transformations the evolution operator is reached (Näpinen and Müürsepp 2002, 468). This is the chain of novelties that leads to the physical-mathematical description with unidimesional time flowing from the past into the future. There are still thinkers who believe that some events are evolving in one direction only because the opposite direction would have a very small probability but is still possible. The view of Ilya Prigogine is different. He considers some states to be strictly forbidden and not possible to be discovered in nature. Thus, irreversibility was made a fundamental property of physics. Time that we are familiar with from classical physics is just an average over his new time operator. Prigogine incorporated reversible and irreversible parts into a new microscopic equation. The equation contains an operator analogous to the Hamiltonian. This is the time evolution operator that can drive the system to both equilibrium or nonequilibrium states.

As a matter of fact, Prigogine does not connect the second law of thermodynamics just with the experimental situation and thermal machines. For him, the scope of the law is much broader. The main idea of the second law is that there is a quantity in nature that changes in only one direction. In that case, he believes, there are also states that change in only one direction. This is a clear novelty of the Prigoginian approach. An analogous interpretation has been presented by Max Planck. The traditional statistical interpretation of the second law of thermodynamics allows movement in the opposite direction as well. It just considers its probability very low. Thus, the Prigoginian interpretation sets a firm theoretical basis for irreversibility. As everything is unique in principle, repeatability of the experiment cannot be 
kept as a strict requirement any more. Nothing can forbid us to repeat experiments of course. We just have to keep in mind that the world out there is irreversible not only in the subjective sense of human sense experience but in the theoretical objective sense as well.

The researcher can still construct the object but the construction will be unique. Repetition of experiments may occur but the "reading" of their results has to change. In order to explain the change in more detail we need to bring in the attractors. The repeatedly achieved result of an experiment often describes a strange attractor. We can never expect to predict where in the attractor the next result will fall. However, we can be for more or less sure that it will not be outside of the attractor. Actually, this is the butterfly generalized. There is repeating but it does not mean that the experiments are any longer repeatable by their essence. The attractor is a new mathematical tool. It enables to describe the behaviour of real complex material systems, like the atmosphere, in a more flexible way than the classical tools like mean and standard deviation. Probably, this flexibility does not give an essentially new material explanation for non-repeatability of experiments. But do we need that. Perhaps a better mathematical tool enabling more precise description is all we can have here as we have the Prigoginian interpretation of the second law of thermodynamics at our disposal already.

Prigogine attempts to have an experimental dialogue with nature rather than finding anything out once and for all. While applying such approach, we need to understand that nature normally says "no" and only very seldom "may be". Still, nature never lies. These are the laws of nature that do so because they are our own creation. How ingenious, is quite an open question.

The lying laws of nature bring us into contact with the thoughts of an influential thinker in philosophy of science today, namely Nancy Cartwright. Her main point is that the laws of physics lie, because they do not accurately describe real situations in the world (Cartwright 1983). Cartwright claims that the physical laws have a fictional character in the sense of literary, theatrical or film fiction. Being the laws of physics, however, demands a unique physical fictive staging.

Nancy Cartwright's central claim concerning the lying laws has been challenged by Joseph Rouse for more than once. At first, Rouse argued that Cartwright's arguments challenge the truth of law-statements only if their meaning were fixed in ways at odds with the actual use of such expressions in scientific practice (Rouse 1987, ch. 5). The literal interpretation of the laws that Cartwright once took to be false does not express accurately what the laws really mean in scientific practice. In a more general interpretation given by Rouse (2008) the experiment appears in a particularly interesting way. The stories of scientific fiction do not necessarily involve experimen- 
tation at all. It is taken for granted as something that is understood well enough. Philosophers have not been too keen about including experimentation into their sphere of interest. Joseph Rouse is correct emphasizing that the logical empiricists, for instance, confined experimentation to the context of justification rather than discovery; post-empiricists and scientific realists emphasized that experimentation presupposes prior theoretical articulation of concepts; while reaction to the excesses of both traditions proclaimed that experimentation has a life of its own apart from developing or testing concepts and theories (Rouse 2008,3 ). Rouse pays a lot of attention to thought experiments in the current context. Our focus, however, will remain with the laboratory.

If we look into the aim of laboratory science we come to realize that experimentation in the laboratory probably has to allow new aspects of the world to show up as conceptually articulable. In the case of classical experimentation this can hardly happen. Here we should rather agree with the logical empiricists. No discovery is possible. It is rather justification we can be looking for. The Prigoginian revolution, however, opens the gate for an ever ongoing experimental dialogue with nature.

In order to understand how new aspects of the world can show up we could take a look at what Ian Hacking has pointed out about how scientists come to know their way around in the world: "In nature there is just complexity, which we are remarkably able to analyse. We do so by distinguishing, in the mind, numerous different laws. We also do so by presenting in the laboratory, pure, isolated phenomena" (Hacking 1983, 226). Obviously, Hacking is speaking about the classical experiment dealing with isolated phenomena. But he is still talking about events in the world rather than appearances to the mind. It is interesting that experimental work was meant not just to strip away confounding complexities to reveal underlying nomic simplicity.

Still, what does it mean to open up a scientific domain and how are such events related to the construction of experimental systems? This is the central question Joseph Rouse puts in his account of laboratory fictions (Rouse $2008,7)$. The latter term asks for an explanation. There is nothing strange about calling thought experiments or theoretical models fictional. But how can the term relate to the most regular laboratory settings? It is an interesting question, especially in the light of the revolution concerning irreversibility. The new type of experimental setting has to be a system consisting of the experimental apparatus as well as an appropriate setting of the mind of the researcher. It is not clear, however, whether Rouse includes the researcher herself into the experimental setting. Rouse speaks of two interconnected features, that of the experimental systems that bring an entire field of phenomena "into the open" or "into the space of reason". First is the systematic 
character of experimental operations. Fictions in this sense are imaginative constructions that have sufficient self-enclosure and internal complexity to constitute a situation whose relevant features can be identified through their mutual interrelations. Second, fictions constitute their own "world" (Rouse $2008,8)$. It is definitely a big step forward in understanding the experiment compared to the classical approach based on just different experimental instances. Fortunately, it is a quite widely recognized attitude in philosophy of science already. It stems back to the Polish thinker Ludwik Fleck (1979) who has also played a big role influencing Thomas Kuhn's conception of the scientific revolutions.

The idea of intentionality and conceptual character of experimental systems asks for an analysis of the constitutive character of laboratory fictions. According to Rouse, the constitution of a scientific domain accounts for the conceptual character of the distinctions functioning within the associated field of scientific work (Rouse 2008, 9). Rouse's account is close to Nancy Cartwright's "blueprints for nomological machines". Still, Rouse has taken a step forward not just by trying to avoid the quite inappropriate machine metaphor but rather by adding conceptually articulable behaviour to just regular behaviour. The whole language of Cartwright is quite inappropriate and technical considering the generalizing trend of making sense of the experimental settings. Both Cartwright and Ronald Giere bring in empirical adequacy or resemblance to "real" systems. Hereby, we agree with Rouse that this move has been made too late. These characteristics would definitely work in classical mechanics, in the field that has set the basis of all modern science but has to be left behind today as a too rough approximation of empirical reality. Today, it is normal for science to progress into new domains of phenomena.

Still, what is the real novelty of Rouse's position in our context of changing the view on the experiment? Is there a qualitative difference from the "classical" views (Cartwright, Giere, etc) or is it just a mastery elaboration? Unfortunately, it seems to be rather the latter that is true. Rouse speaks about parallel development of practical and verbal articulation as well as development and refinement of instruments (Rouse 2008, 13). This is useful of course. But it can hardly give us a new understanding of the experiment.

But the general trend is still very promising. Looking into Hasok Chang's (2004, 59-60) considerations on measurement supports the idea. Chang calls the case "the problem of nomic measurement". This is when we identify a concept $\mathrm{X}$ by some other phenomenon $\mathrm{Y}$. It is interesting that the issue does not apply only to quantitative measurement. First of all it is a problem of the projection of the concept to be right or wrong. Such type of measurement affects non-quantitative concepts as much as it applies to measurable quan- 
tities. Here we probably have an elaboration of what Henri Poincaré and René Thom were suggesting while endorsing the qualitative approach over the quantitative one. The experimental studies of Prigogine are clearly consistent with these ideas. Research into the nature of the so-called dissipative structures can be made quantitative as far as the results are concerned. Such numerical results, however, would not make any sense whatsoever without qualitative explanations. Sometimes they support the qualitative interpretation. Sometimes it is just the qualitative pattern we can reasonably be interested in.

After all, it is not just Prigogine, or Thom or Poincaré. It is not true that it is always the qualitative result that we are aiming at? Even if it has a purely quantitative empirical value it is still the interpretation that stems from it that we are really interested in. If an athlete is doing high jump it is not just the result itself she achieves that interests us. We are rather interested whether anyone jumped higher at that particular competition and whether the result is unique in some respect, a world record or perhaps just the personal best. Needless to say, we often still need the quantitative value in order to be able to give the qualitative interpretation. Interestingly enough, the opposite can be the case as well. Many physical phenomena turn out to depend on the qualitative properties of the laws that control or describe them. This means that the same basic events may occur over a large variety of quantitative data. The events occurring cannot be exactly the same of course but this is not needed from the perspective of the qualitative approach.

Laying the stress on the qualitative side of research contributes to the survival of the experiment. Both can be OK, having just one result and giving it qualitative interpretation or repeating the experiment with diverging results that describe a strange attractor.

\section{The Maxwellian Revolution}

We have explained the Prigoginian revolution. It has a direct impact on the experimental situation in science. Prigogine aims at a constant experimental dialogue with nature adhering to the arrow of time that makes the material experiment non-repeatable by definition.

Let us now take a brief look at the Maxwellian revolution. This is Nicholas Maxwell whose ideas we are going to address, not James Clerk. Nicholas Maxwell advocates the switch from knowledge-inquiry to wisdom-inquiry (Maxwell 2010). This is a call for redefinition of the essence of science that cannot leave the role of the experiment unaffected. Maxwell has declared the scientific reality based on physical research alone too narrow. His idea is making the social sciences and humanities the real foundation of scientific inquiry, shifting its aim at wisdom rather than knowledge. This is really a 
revolutionary view as it implies the strife for modelling the physical sciences after humanities and social sciences. Looks an impossible task and perhaps is that from the point of view of the methodology of classical science. What Maxwell really means, however, is bringing natural sciences into the context of humanities in the sense that they should serve humanity directly or at least constantly keep this task in mind. The experiment is never on the foreground of Maxwell's approach. However, his philosophy of science can be understood as a version of practical realism as he clearly acknowledges at least four of the five main theses of practical realism (Vihalemm 2011, 57).

In the light of the Maxwellian revolution the role of the experiment in science becomes relatively marginal. It is entitled to reduce to just one component of research into nature and perhaps not the most important one. However, it will definitely still have its role to play. Knowledge pursuit remains an important stage of the structure of wisdom. At least in chemistry, one can hardly imagine the pursuit to have any success without the experiment, although probably the new non-repeatable one, at work.

Maxwell has not paid special attention to the experiment. The role of the latter, however, will necessarily be subjected to change if wisdom-inquiry becomes implemented. Most valuable knowledge will not be produced by experimental science any more. Rather the opposite will be the case. The whole wisdom-inquiry has to be aimed at knowledge that is not based on experimental natural science any more by definition.

\section{Conclusion. The Future of the Experiment}

Just like the past of the experiment, although not scientific, the future seems to be with chemistry. Experimentation became part and parcel of human culture in the era of alchemy. Still, this was not scientific experimentation in the modern sense. The latter was started by Galileo who was able to propose testable hypotheses contrary to the spurious experimentation of the alchemists where no reasonable hypothesis was present. Thus, physics became the model of the whole science, the experimental one included.

What is the situation like today? Physics in its classical form has practically ceased to exist. The experiment in physics has turned into something quite specific compared to the Galilean era. A large part of physics as fundamental science has become nonexperimental in the classical sense. The measures involved in research simply cannot be reached by human senses even with the help of amplifying devices like the telescope or microscope. It is just the mathematical, perhaps partly even metaphysical discipline that has been left over. Still, there is applied research in physics where the experiment is essential. The future of the experimental science, however, seems to be with chemistry. If we look at the most typical trends of chemical research 
we see only irreversible processes. Obviously, one can repeat an experiment. The result will not be the same. But what we can study is whether the process remains in the limit of the same (strange) attractor. ${ }^{1}$

In conclusion, both of the following positions can be accepted. One can claim that there is no basic difference in how we understand the experiment today and in the past. Although we cannot repeat it with exactly the same results, their convergence is enough to make the experiment successful. It does not matter, whether we call the supporting structure structural stability or apply the concept of the strange attractor. The others can say that there is a big difference. Not just a practical one but caused by the theoretically justified irreversibility in the material world out there.

There are two types of sciences, old and new. The old classical one is history. Therefore, the same applies to repeatable experiments. Today, we need to live with irreversibility and non-repeatability that bring about uncertainty. There seems to be at least some ground for the Maxwellian revolution in place. Natural science is approaching the humanities and social research at least partly as the result of the changing role of the experiment. In a more general sense, however, the change is guided by the arrow of time, the evolutionary approach to both natural and social science.

\section{Acknowledgements}

Completion of the final version of the paper was supported by the Estonian Science Foundation Grant No. 9362.

\section{Bibliography}

Cartwright, N. (1983). How the Laws of Physics Lie, Oxford University Press, Oxford.

Chang, H. (2004). Inventing Temperature, Oxford University Press, Oxford.

Fleck, L. (1979). Genesis and Development of a Scientific Fact, University of Chicago Press, Chicago.

Hacking, I. (1983). Representing and Intervening, Cambridge University Press, Cambridge.

1 There are numerous examples of such experiments at the Steve Spangler website which is actually meant for making science exciting for children. But these examples also perfectly illustrate the essentially irreversible nature of the experiment of today. Milk always changes colour in a somewhat different way. However, the colour always changes. 
Maxwell, N. (2010). Cutting God in Half-And Putting the Pieces Together Again. A New Approach to Philosophy, Pentire Press, London.

Müürsepp, P. (2010). Structural Stability as the Core of René Thom's Philosophy, Lambert Academic Publishers.

Näpinen, L. and Müürsepp, P. (2002). The concept of chaos in contemporary science: On Jean Bricmont's critique of Ilya Prigogine's ideas, Foundations of Science 7: 465-479.

Prigogine, I. (1997). The End of Certainty, The Free Press, New York.

Rouse, J. (1987). Knowledge and Power, Cornell University Press, Ithaca.

Rouse, J. (2008). Laboratory fictions, in M. Suarez (ed.), Fiction in Science: Philosophical Essays on Modeling and Idealization, Routledge, New York, pp. 37-55.

Thom, R. (1975). Structural stability and Morphogenesis, W. A. Benjamin, Inc, New York.

Vihalemm, R. (2007). Philosophy of chemistry and the image of science, Foundations of Science 12: 223-234.

Vihalemm, R. (2011). Towards a practical realist philosophy of science, Baltic Journal of European Studies 1: 46-6o.

Walker, I. (1983). Complex-irreversibility and evolution, Cellular and Molecular Life Sciences 8: 806-813. 